\title{
Factors Regulating the Reversibility of Long-Term Potentiation
}

\author{
Ursula Stäubli and Daniel Chun \\ Center for Neural Science, New York University, New York, New York 10003
}

Theta burst stimulation (TBS) produces an extremely stable form of long-term potentiation (LTP). In contrast, a brief episode of single-pulse stimulation at theta frequency [theta pulse stimulation (TPS)] has been demonstrated to reverse LTP in area CA1 of slices and freely moving animals without causing depression when administered to nonpotentiated pathways. The present in vitro studies confirm these results and establish that the susceptibility of LTP to reversal and the degree of depotentiation are time-dependent. Specifically, a 1 min train of TPS delivered 30 sec after LTP induction produced an almost complete and lasting depotentiation but had increasingly less impact at longer delays and virtually no effect at 30 min. Increasing the duration of TPS did not cause more depotentiation. However, pharmacological facilitation of AMPA receptormodiated currents significantly enhanced the strength of the reversal process and allowed TPS to produce robust depotentiation up to 30 min after LTP induction. The reversal effect was selective to potentiated synapses receiving TPS, was reversible, and was not blocked by NMDA receptor antagonism. Additional experiments indicated that AMPA receptor facilitation promotes depotentiation by enhancing an active process triggered by TPS that reverses expression without extending the time course of consolidation. These results suggest that the mechanisms responsible for LTP reversal are linked to the amplitude and/or duration of fast excitatory currents mediated by synaptic AMPA receptors and are effective up to the completion of LTP stabilization.

Key words: hippocampus; CA1; reversal of LTP; depotentiation; theta bursts; theta pulses; AMPA receptor facilitation; NMDA receptor antagonism; consolidation period
In recent years, the idea of a depressive counterpart to long-term potentiation (LTP) has become increasingly popular, partly because of questions regarding saturation of memory and possible causes for loss of memory and partly because weakening of synapses has considerable theoretical significance in learning algorithms of neuronal network models. Early tests of the synapse specificity of LTP in arca CA1 indicated that although LTP was specific to the tetanized pathway, it was associated with depression of synaptic transmission in a converging untetanized pathway (Andersen et al., 1977; Lynch et al., 1977; Dunwiddie and Lynch, 1978). Such heterosynaptic depression, however, is not a necessary concomitant of LTP and, in fact, is elicited best by traitus that do not produce LTP, whereas stimulation with the optimal parameters for I.TP does not alter the synaptic strength of neighboring, nonstimulated connections (Dunwiddie and Lynch, 1978; Larson and Lynch, 1986).

Recently, much effort has been directed at exploring a phenom enon called long-term depression (LTD), a form of homosynaptic depression produced by repetitive low-frequency activation $(1 \mathrm{~Hz})$ of excitatory inputs to hippocampus and other areas (for review, sec Bear and Malenka, 1991). Although this work primarily focuses on depression of naive pathways, a parallel line of research is concerned with the selcctive depression of potentiated inputs called depotentiation, a phenomenon produced by low-frequency activation of the same population of fibers that was tetanized previously. Behavioral studies on the stages underlying memory formation have shown that various manipulations can disrupt the cncoding of information if applied shortly after initial learning,

Received July 31, 1995; revised Oct. 10, 1995; accepted Oct. 16, 1995.

This research was supported in part by a grant from the whitehall Foundation (F93-17) to U.S

Correspondence should be addressed to Dr. Ursula Stăubli, New York University, Center for Neutal Science, 4 Washington Placc, New York, NY 10003.

Copyright 91996 Society for Neuroseience 0270-6474/96/160853-08\$05.00/0 thereby causing retrograde amnesia (McGaugh, 1966; McGaugh et al., 1993). By analogy, LTP appears to pass through a consolidation period during which it is susceptible to disruption. For example, brief periods of hypoxia and application of adenosine receptor agonists reverse LTP in vitro when administered within 1-3 min of induction, but not thereafter (Arai et at., 1990a,b). Work done several years ago indicated that LTP can be reversed in anesthetized rats by low-frequency stimulation at $1 \mathrm{~Hz}$ applied within minutes of induction, whereas the same stimulation administered to naive synapses was ineffective (Barrionuevo et al., 1980). These results were confirmed in area CA1 of freely moving animals using brief, low-frequency stimulation at $1-5 \mathrm{~Hz}$ (Stäubli and Lynch, 1990). Subsequent in vitro findings demonstrated that depotentiation can be produced reliably with stimulation pulses paced at theta frequency $(5 \mathrm{~Hz})$ delivered shortly after LTP induction, whereas $1 \mathrm{~Hz}$ low-frequency stimulation did not reverse LTP and $10 \mathrm{~Hz}$ stimulation was no more effective than $5 \mathrm{~Hz}$ (Larson et al., 1993). That theta burst stimulation (TBS) and theta pulse stimulation (TPS) are optimal for inducing and reversing LTP, respectively, is intriguing considering that hippocampal cells in behaving rats tend to fire in single spikes or short bursts, both synchronized to the theta rhythm (Hill, 1978; Otto et al., 1991).

The present study was aimed at determining the factors controlling the reversibility of LTP triggered by a physiologically realistic stimulation pattern. First, the time dependence of the reversal effect produced by TPS was investigated, an important issue that never had been examined formally. A second issue concerned the extent to which the reversal process might be linked to AMPA receptor-mediated mechanisms. To test this, an analog of 1-(1,3-benzodioxol-5yl-carbonyl)piperidine (BDP-12) was used, a member of the pyrrolidinone family of compounds shown to enhance selectively currents mediated by the AMPA 
receptor (Arai et al., 1994) and thereby promote LTP induction (Stäubli et al., 1994a).

\section{MATERIALS AND METHODS}

Experiments were conducted on hippocampal slices prepared from male Sprague-Dawley rats weighing $180-360 \mathrm{gm}$. The rats were decapitated, and their brains were removed rapidly and placed in $0^{\circ} \mathrm{C}$ oxygenated (95\% $0_{2} / 5 \% \mathrm{CO}_{2}$ ) artificial CSF (aCSF) of the following composition (in mM): $\mathrm{NaCl} 124, \mathrm{KCl} 3, \mathrm{KI}_{2} \mathrm{PO}_{4} 1.25, \mathrm{MgSO}_{4} 2.5, \mathrm{CaCl}_{2} 3.4, \mathrm{NaHCO}_{3} 26$, D-glucose 10 , and L-ascorbate 2 . One of the hippocampi was quickly dissected free in ice-cold aCSF and placed on a Mcllwain tissue chopper in which its middle portion was cut into $400 \mu \mathrm{m}$ sections and collected into a Petri dish containing ice-cold aCSF. From there, selected slices were placed immediately on a nylon net in an interface chamber and maintained at $31 \pm 1^{\circ} \mathrm{C}$. The slices were perfused continuously with aCSF at a rate of $75 \mathrm{ml} / \mathrm{hr}$, and the upper surface was exposed to warm, humidified $95 \% \mathrm{O}_{2} / 5 \% \mathrm{CO}_{2}$. Recording began after a $1 \mathrm{hr}$ incubation.

Population (field) EPSPs were measured with a glass micropipette filled with $2 \mathrm{~mm} \mathrm{NaCl}$ and positioned in the middle of stratum radiatum of field CA1b. Two bipolar stimulating electrodes (twisted $65 \mu \mathrm{m}$ strands of nichrome wire) were placed in equivalent positions of CA1a and CA1c, respectively, to activate separate (nonoverlapping) populations of Schaffer collateral-commissural (S-C) fibers. Their positions were arranged so that the same amount of current evoked two responses that did not differ from each other by $>10 \%$. The stimulus strength of the test pulses was adjusted to produce a response for which the amplitude was $\sim 60 \%$ of the maximum spike-free response. Stimulation pulses were alternated between the two pathways at intervals of $10-20 \mathrm{sec}$ such that each pathway received a pulse every $20-40 \mathrm{sec}$. The recording signals were digitized by computer, which calculated the slope and amplitude on-line and stored the data to disk. If slope and amplitude of the field EPSPs did not fluctuate by $>10 \%$ during a $15-20 \mathrm{~min}$ control period, the responses were used as baseline values before experimental tests. LTP always was induced using TBS consisting of ten 30 -msec-long bursts with four pulses at $100 \mathrm{~Hz}$ each, separated by $200 \mathrm{msec}$. The stimulation intensity was increased to trigger a spike component in the dendritic field potential, usually by doubling the pulsc duration. This stimulation pattern has been shown to be optimal for producing saturated levels of LTP (Larson et al., 1986). Testing with single pulses then was resumed to confirm that LTP had been established. Attempts to reverse LTP were conducted with TPS and consisted of a $1 \mathrm{~min}$ episode of single-pulse stimulation with the pulses separated by $200 \mathrm{msec}$. In one set of experiments, a 3 min TPS train was used. The stimulation intensity was not increased during TPS.

The drug solution containing AMPA receptor modulator was prepared fresh daily by dissolving BDP-12 (Cortex Pharmaceuticals, Irvine, CA) in to aCSF and injecting it into the medium with a perfusion pump. Care was taken to keep the total rate of infusion into the chamber constant throughout the experiment $(75 \mathrm{ml} / \mathrm{hr}$ ) by adjusting the flow rate of the primary source of aCSF accordingly. In some experiments, the NMDA receptor antagonist D,L-aminophosphonopentanoic acid (AP-5; $200 \mu \mathrm{M}$ ) was dissolved in aCSF and added immediatcly after LTP induction with a second syringe pump.

\section{RESULTS}

In all of our experiments, TBS reliably produced robust LTP. The results from the first set of experiments confirmed and cxtcnded earlier findings showing that stable LTP in area CA1 of freely moving animals (Stäubli and Lynch, 1987) can be reversed with low-frequency stimulation at $5 \mathrm{~Hz}$ (Stäubli and Lynch, 1990). In addition, the present results establish that the magnitude of the depotentiation effect is time-dependent and inversely related to the length of the delay between TBS and TPS. As illustrated in Figure 1, a 1 min train of TPS was sufficient to reverse LTP almost completely if delivered $30 \mathrm{sec}$ after induction but had increasingly less impact when given at longer intervals. Reversal attempts conducted between $30 \mathrm{sec}$ and $15 \mathrm{~min}$ all produced a significant amount of depotentiation as revealed by the paired two-tailed $t$ test $\left(30 \mathrm{sec}: t_{(5)}=10.23, p<0.0002 ; 3 \mathrm{~min}: t_{(5)}=6.82, p<0.001\right.$; 6 min: $t_{(5)}=5.37, p<0.003 ; 10$ min: $t_{(4)}=5.05, p<0.007 ; 15 \mathrm{~min}$ : $t_{(4)}=4.55, p<0.02$ ). At a delay of $30 \mathrm{~min}$ or longer, the mean of the paired differences between the LTP measured before and 45 min after reversal attempts was $<10 \%$, a decrement considered too modest to be attributable only to the action of an active reversal process as opposed to an intrinsic decay of potentiation.

Attempts to increase the amount of depotentiation by applying a prolonged train of TPS failed. That is, the average degree of reversal obtained with a 3 min train of I'PS delivered $30 \mathrm{sec}$ after LTP induction was $79.9 \pm 4.9 \%(n=4)$ compared with $84.3 \pm$ $5.3 \%(n=6)$ obtained with a 1 min TPS episode; when delivered 15 min after LTP induction, the mean depotentiation obtained with a 3 min train was $27 \pm 8.1 \%(n=5)$ compared with $19.0 \pm$ $3.0 \%(n=5)$ produced by a 1 min TPS episode.

The drug BDP-12, when added to the perfusion medium, was found to strengthen the reversal process and thereby prolong the time period during which TPS produced a significant amount of depotentiation. As illustrated in Figure $2 A$, in these experiments reversal attempts always occurred $15 \mathrm{~min}$ after LTP induction, first in one pathway and then in the presence of BDP-12 in the second pathway, and the amount of LTP remaining 45 min after TPS was compared between control and test input within the same slice. Without the drug present, TPS at 15 min caused a small amount of LTP reversal, which is in agreement with the results obtained in the first set of experiments (Fig. 1). In contrast, TPS delivered $15 \mathrm{~min}$ after induction in the presence of $500 \mu \mathrm{M}$ BDP-12 produced an almost complete reversal of LTP in the test pathway (see Fig. 2A). Consistent with results from previous studies (Stäubli et al., 1994a,b), pharmacological facilitation of AMPA receptors increased the amplitude and, to a lesser degree, the slope of baseline synaptic responses and significantly enhanced their duration, effects that usually reached a plateau after 10-15 min; also, TBS delivered in the presence of the drug produced robust LTP. Group data illustrating the dose-dependent facilitatory action of BDP-12 on the magnitude of depotentiation are shown in Figure $2 B$. At concentrations between 250 and 500 $\mu \mathrm{M}$, the mean depotentiation was significantly larger than that obtained when TPS was given to the control input of the same slice $\left(t_{(7)}=6.67, p<0.0003\right)$. The average LTP reversal obtained with BDP-12 at a dose of $100 \mu \mathrm{M}$, but not $50 \mu \mathrm{M}$, was still significantly greater than that measured for control inputs stimulated without the $\operatorname{drug}\left(t_{(3)}=9.99, p<0.002\right)$.

Subsequent experiments demonstrated that the facilitatory action of BDP-12 on LTP reversal was time-dependent. As illustrated in Figure $3 A$, the magnitude of depotentiation declined successively as the delay between TBS and TPS increased from 15 to 30 to 45 and, finally, to $60 \mathrm{~min}$. Group data are shown in Figure $3, B$ and $C^{\prime}$, and were analyzed by comparing the magnitude of potentiation remaining $45 \mathrm{~min}$ after TPS with the LTP level mcasured before TPS. The results revealed a significant degree of depotentiation where reversal attempts occurred 15 and $30 \mathrm{~min}$, but not 45 and $60 \mathrm{~min}$, after LTP induction (15 min: $t_{(8)}=8.09, p$ $<0.0001 ; 30$ min: $t_{(4)}=3.45, p<0.03$; paired two-tailed $t$ test). Comparisons also were made between the amount of depotentiation obtained with and without the drug (see Fig. $3 C^{\prime \prime}$ ): TPS at 15 or 30 min produced a significantly larger degree of depotentiation than when delivered in the absence of BDP-12 (15 min: $t_{(11)}=$ $5.39, p<0.0002 ; 30$ min: $t_{(7)}=1.96, p<0.05$; one-tailed unpaired $t$ test).

In all of the initial studies, BDP-12 was added before LTP induction. It was possible, therefore, that the drug altered the events occurring during theta burst activity that trigger stabilization chemistries, resulting in a slower consolidation of potentiation. Under such conditions, a normal reversal process would be effective over a longer than normal postinduction period. There- 
A
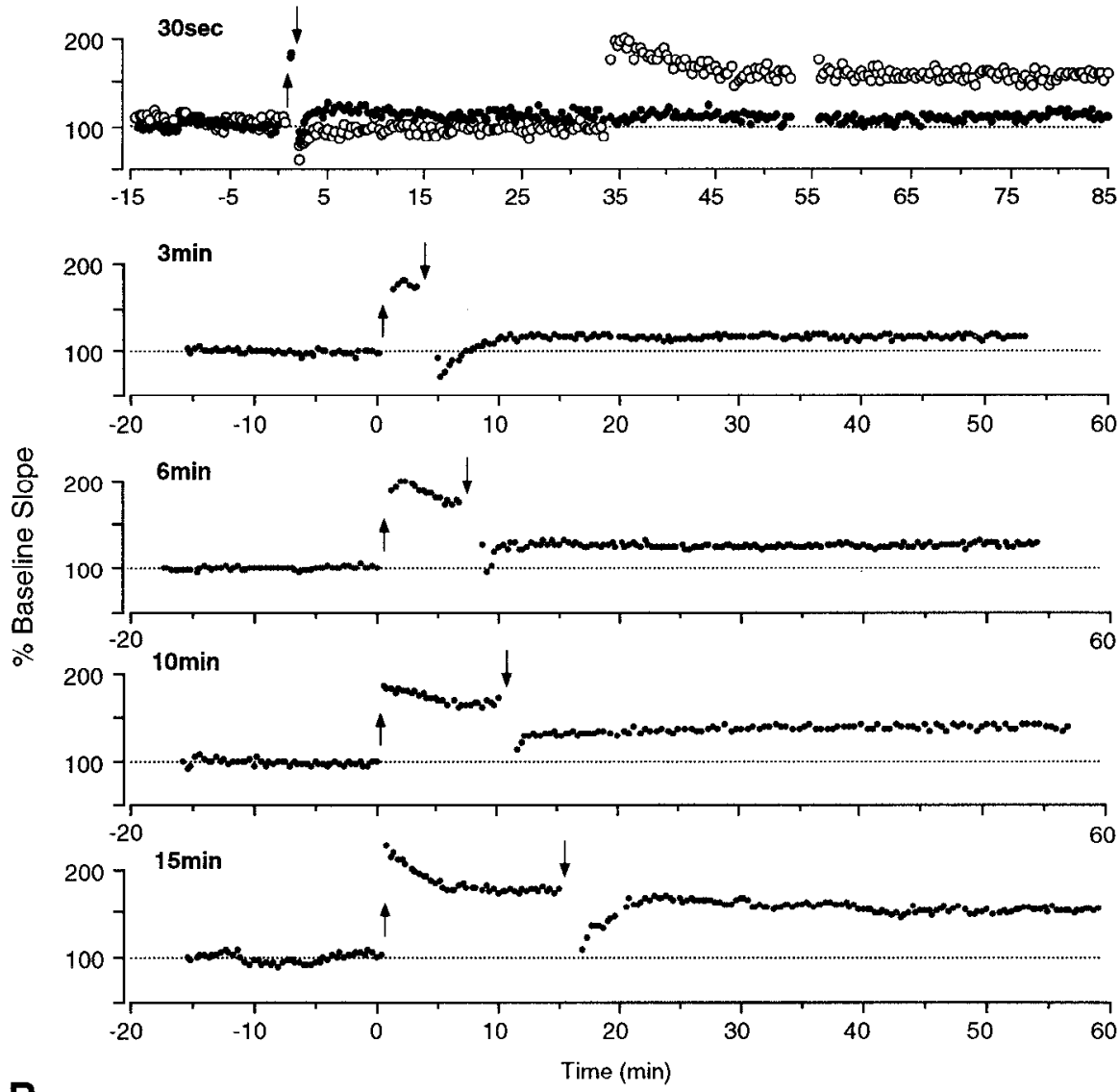

B

$\square$ before TPS

$45 \mathrm{~min}$ after TPS
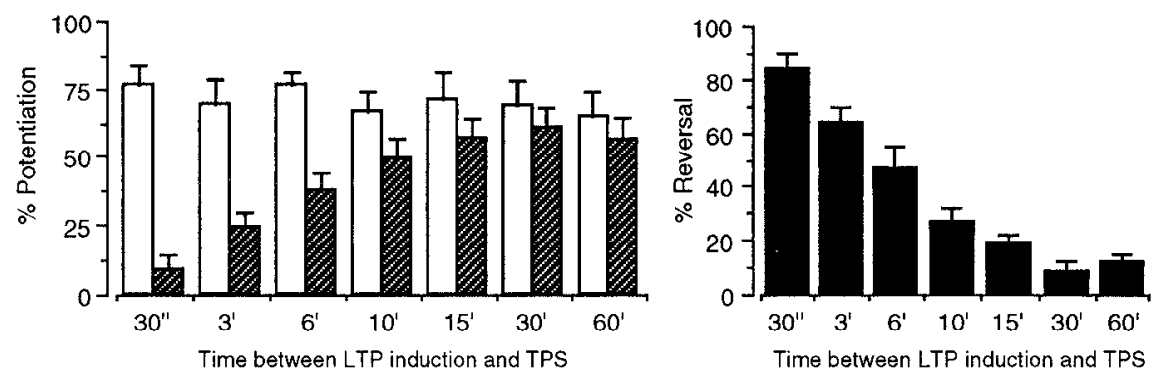

Figure 1. Time dependence of LTP reversal. A, Experiments in which two independent pathways synapsing on the same population of dendrites were stimulated alternately. Shown are individual representative experiments in which reversal attempts involved a 1 min train of TPS given at increasingly longer delays after LTP induction in the test pathway. Subsequent induction of LTP in the control pathway was used to verify that the slices were healthy and able to sustain LTP (example shown in experiment at top). In all of the figures, TBS is represented with an upward arrow and TPS is represented with a downward arrow. B: Left bar graph, Group data (mean \pm SEM) illustrate the average LTP measured immediately before and 45 min after reversal attempts made at different delays ( $n=6$ at $30 \mathrm{sec} ; n=6$ at $3 \mathrm{~min} ; n=6$ at $6 \mathrm{~min} ; n=5$ at $10 \mathrm{~min}, n=5$ at $15 \min ; n=4$ at $30 \mathrm{~min} ; n=4$ at $60 \mathrm{~min}$ ). The percent potentiation represents the average LTP calculated from values collected over a 5 min period (except for the 30 sec and 3 min experiments). Right bar graph, Same data as shown on left but expressed as magnitude of depotentiation.

fore, to determine whether the drug facilitates the reversal mechanism per se rather than delays consolidation, BDP-12 (500 $\mu \mathrm{M})$ was added after LTP induction and reversal attempts followed 15 min later. In these experiments, LTP was induced in both pathways simultaneously, but TPS was given only to the test input to assess whether the reversal effect was restricted to the population of potentiated synapses that received TPS. As illustrated in Figure $4 A$, no long-lasting heterosynaptic effects were observed with TPS, i.e., the potentiated control input did not lose its potentiation, whereas LTP in the test input was reduced markedly and significantly $\left(t_{(5)}=8.62, p<0.0003\right.$; paired two-tailed $t$ test). Figure $4 B$ shows that the facilitatory action of the drug on reversal was comparable despite the presence or absence of BDP-12 during theta burst activity.

To test the possibility that the LTP reversal was attributable to deterioration of individual synapses rather than to a true decrease in synaptic efficacy, attempts were made to reinduce LTP after a reversal. An example of such an experiment and group data are shown in Figure 5. After reversing LTP and washout of the drug $(500 \mu \mathrm{M})$, a second TBS treatment reinstated the potentiation. 
A
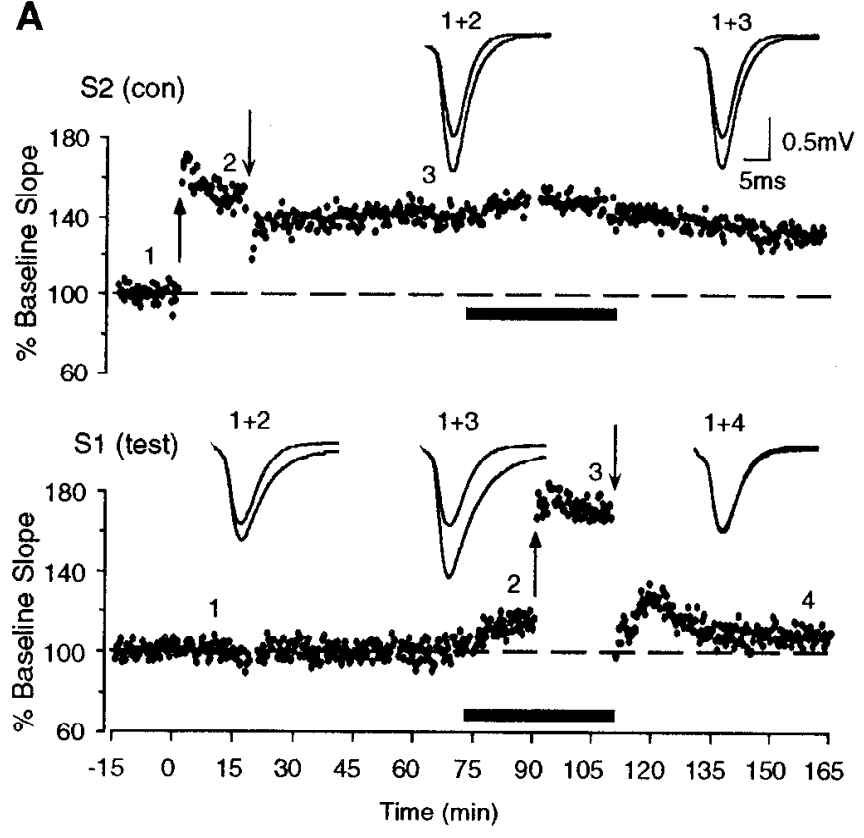

B

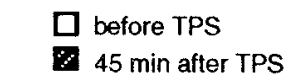

Test Pathway
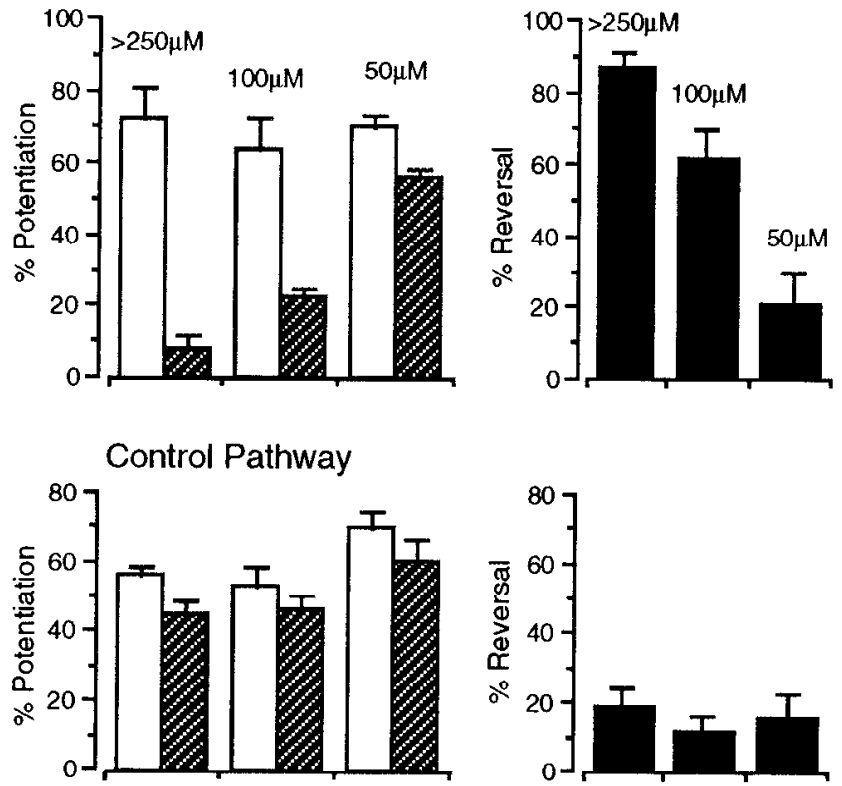

Figure 2. AMPA receptor modulator facilitates LTP reversal in a dosedependent manner. $A$, LTP first was induced in the control input and was followed by an attempt to reverse the potentiation 15 min later with a 1 min episode of TPS. The test pathway received the same stimulation treatment but in the presence of $500 \mu \mathrm{M}$ BDP-12 (black horizontal bar). TPS had no heterosynaptic effects on LTP in the control pathway but almost completely reversed LTP in the test pathway. Insets show superimposed EPSPs taken at the times indicated above each graph. B: Top, Group data for the test pathway illustrate, in the left graph, the amount of LTP before and $45 \mathrm{~min}$ after reversal attempts occurring $15 \mathrm{~min}$ after induction and, in the right graph, the magnitude of the resulting depotentiation produced in the presence of different concentrations of BDP-12 ( $n$ $=8$ at $>250 \mu \mathrm{M} ; n=4$ at $100 \mu \mathrm{M} ; n=5$ at $50 \mu \mathrm{M}$ ). The amount of potentiation was calculated as the average of the values over a $5 \mathrm{~min}$ period. B: Bottom, Group data for the second input (control pathway) in the same slices as above, except that induction of LTP and reversal occurred without the drug present.
Because the AMPA rcceptor modulator itself contributed to the enhanced response during the first, but not the second, LTP episode, the potentiation measured before the reversal was slightly larger.

Additional experiments $(n=3)$ tested whether TPS delivered to a naive pathway in the presence of BDP-12 $(500 \mu \mathrm{M})$ would lead to synaptic depression. A typical experiment of this kind is illustrated in Figure $5 B$, which shows that TPS caused an initial temporary depression of the response followed by a recovery to the predrug baseline level. The failure of TPS to induce LTD-like effects was re-examined in five additional slices in which BDP-12 was left in the medium throughout the entire experiment. The results confirmed that naive responses are immune to TPS; presence of the drug for $15 \mathrm{~min}$ increased the slope of the response to a plateau of $111 \pm 1 \%$, and TPS caused a depression to $101 \pm 1 \%$ during the first $5 \mathrm{~min}$ followed by a recovery of the response size to the original drug baseline level $(110+3 \%$ at $30 \mathrm{~min}$ after TPS; data not shown).

Assessing whether TPS produces a reversal of LTP via activation of NMDA receptors requires application of AP-5 in sufficient concentration within the time window of vulnerability. The discovery that BDP-12 strengthens the reversal process such that TPS at 15 min after LTP induction still produces a significant degree of depotentiation enabled us to test whether activation of NMDA receptors is critical for the reversal to occur. As illustrated in Figurc 6, LTP was induced in the presence of $500 \mu \mathrm{M}$ BDP-12 and was followed by infusion of $200 \mu \mathrm{M}$ D,L-AP- 5 and attempts to reverse LTP 15 min later. In each of the five experiments conducted, the NMDA receptor antagonist was unable to prevent the reversal. The average amount of depotentiation was $91.4 \pm 4.4 \%$, which is virtually identical to that obtained in eight slices that were given TPS without AP-5 present $(87.5 \pm 3.7 \%$; see Fig. $2 B,>250 \mu \mathrm{M})$. That the concentration of AP-5 was sufficient to suppress LTP was verified by showing that TBS delivered to the control input in the presence of AP-5 did not produce LTP (data not shown).

\section{DISCUSSION}

The above results confirm that different modes of cell activation (bursts and single spikes) modulated by an endogenous rhythm (theta) can either induce LTP or reverse it. Considering that bursts and spikes, both synchronized to the theta rhythm, are common firing patterns observed in pyramidal cells of rats during exploration, it is possible that induction and reversal occur during behavior.

The present experiments establish in detail the time dependence that governs the LTP reversal phenomenon. The finding that TPS was effective only within a limited time after LTP induction suggests that the stimulation actually reversed the changes that subserve LTP, rather than being merely a disruptive process. In support of this interpretation are the following observations. (1) TPS returned the potentiated responses to values above or near their baseline levels, and not beyond, as expected if the depotentiation was the result of an extrasynaptic effect. (2) TPS had no impact when delivered to naive synapses, and when delivered to potentiated synapses, it did not cause heterosynaptic LTP reversal in a sccond potentiated input within the same slice, as might occur if the stimulation caused a general depression. (3) After LTP reversal, the response could be repotentiated to its original strength.

The mechanisms responsible for the depotentiation effect are not clear. Physiological studies indicate that the bursts of afferent stimulation used to induce LTP initiate two types of processes, 

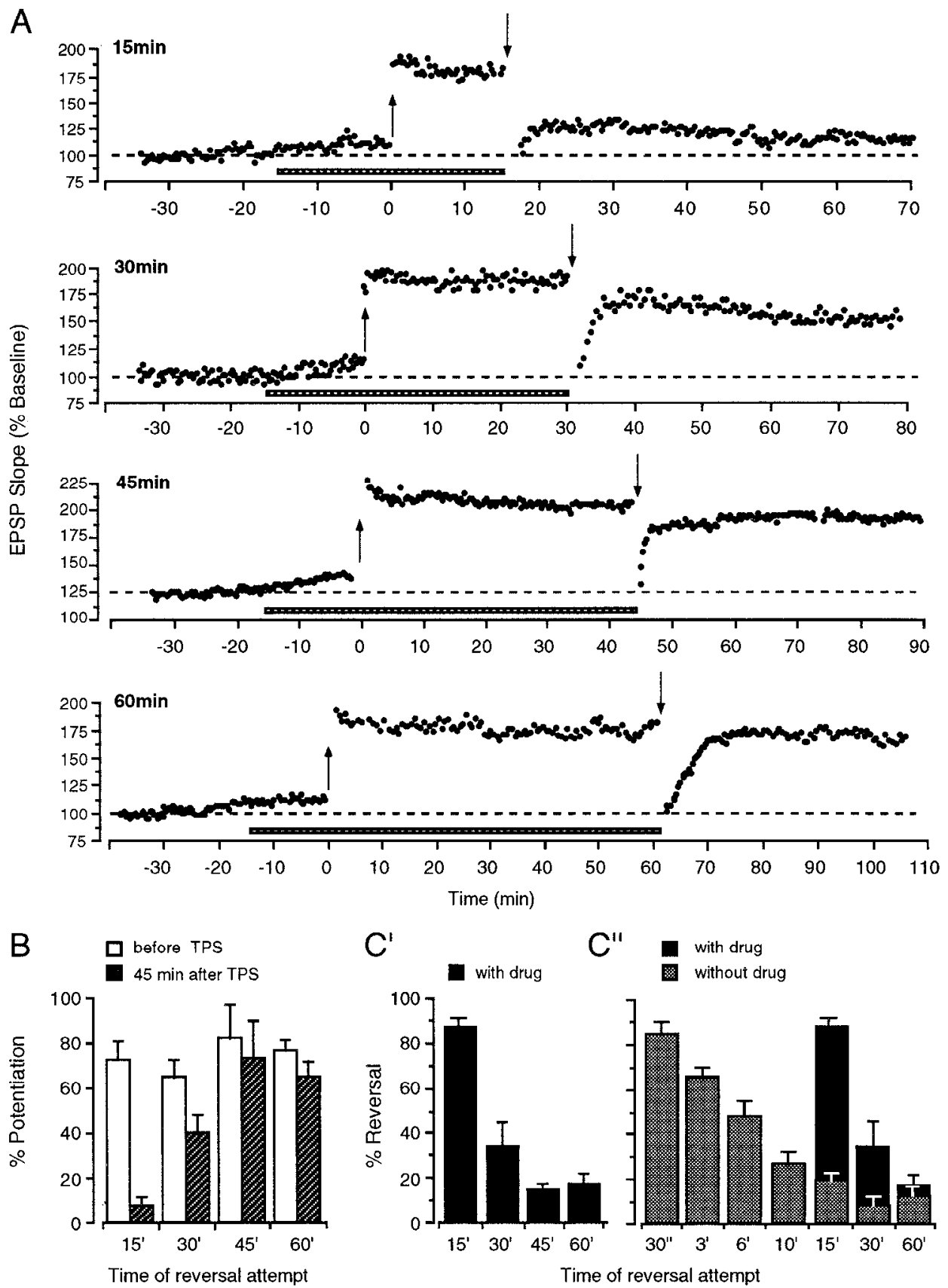

Figure 3. AMPA receptor modulator facilitates LTP reversal in a time-dependent manner. A, Individual representative experiments in which LTP induction and reversal attempts were conducted in the presence of BDP-12 (>250 $\mu \mathrm{M}$; hatched horizontal bar). TPS was administered at increasing delays. $B$, Group data of experiments conducted in the presence of BDP-12 show the amount of LTP measured immediately before and 45 min after reversal attempts made at different delays, i.e., at $15 \min (n=8), 30 \mathrm{~min}(n=5), 45 \mathrm{~min}(n=4)$, and $60 \mathrm{~min}(n=3)$. The percent potentiation represents the average LTP calculated from values collected over a 5 min period. $C^{\prime}$, Same data as in $B$ but expressed as magnitude of depotentiation. $C^{\prime \prime}$, Amount of depotentiation obtained in the presence of the drug (black bars) compared with the results obtained without the drug (superimposed gray bars) (see Fig. $1 B)$ at different delays.

i.e., those leading to the expression of potentiation and those responsible for its consolidation. The first appears to require 20-30 sec (Gustafsson et al., 1989), whereas the second takes an unknown number of minutes to reach completion. The finding that pharmacological modulation of AMPA receptors by BDP-12 promotes the reversal of LTP, irrespective of whether the drug is added before or after TBS, is crucial. It suggests that facilitation of AMPA receptors enhances an active process in the postinduction period and does not alter events occurring during theta burst activity, thereby delaying the time course of stabilization processes set in motion during LTP induction. Presumably then, BDP-12 enhances the effectiveness of an actual depotentiation mechanism (triggered by TPS) that reverses consolidation events and is effective up to the completion of stabilization. According to this argument, consolidation of LTP generally takes considerable time to develop (at least $30 \mathrm{~min}$ ), more than was inferred originally from previous studies (Arai et al., 1990a,b; Larson et al., 1993). In summary, the above results imply that (1) the drug made the 
A
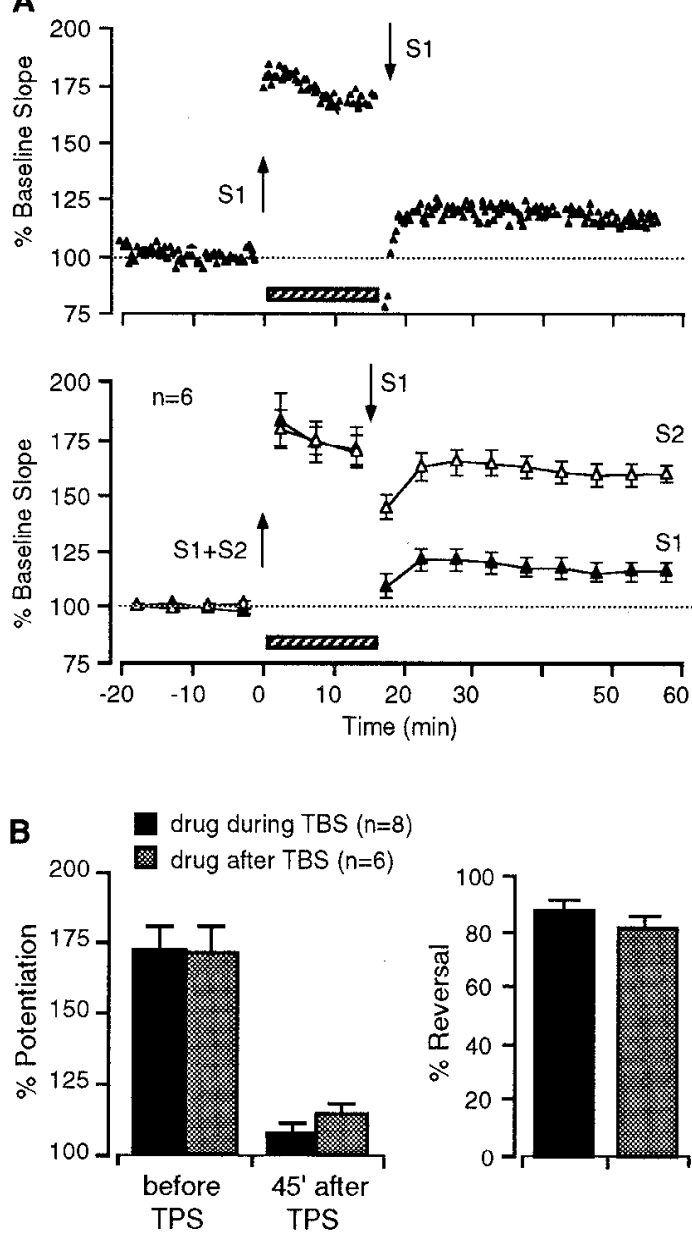

Figure 4. AMPA receptor modulator facilitates depotentiation by enhancing a reversal process in the postinduction period. $A$, Individual representative example of a series of experiments (top) and group data (bottom) testing whether BDP-12 maintains the ability to promote LTP reversal when administered after LTP induction. In all of these experiments, LTP was induced simultaneously in the test (S1) input and control $(S 2)$ input. The drug $(250-500 \mu \mathrm{M})$ (hatched horizontal bar) was added immediately after induction, followed $15 \mathrm{~min}$ later by 'I'PS' to the test pathway (S1) alone. B: Left bar graph, Group data compare the amount of LTP measured immediately before and 45 min after reversal attempts among the six experiments in which the drug was given after TBS (gray bars) and experiments in which the drug was added before LTP induction (black bars) (same data as in Fig. $2 B:>250 \mu \mathrm{M}$ group). Right har graph, Same data as at left but expressed as magnitude of depotentiation.

reversal process itself more effective without prolonging the vulnerable phase directly, and (2) the strength of the reversal process is linked in some way to the magnitude or duration of fast excitatory currents mediated by synaptic AMPA receptors.

Only recently has the phenomenon of LTP reversal become the focus of considerable attention, as indicated by an increased number of reports over the past few years (Barrionuevo et al., 1980; Stäubli and Lynch, 1990; Fujii et al., 1991, 1992; Bittar and Muller, 1993; Larson et al., 1993; Wexler and Stanton, 1993; Bashir and Collingride, 1994; O'Dell and Kandel, 1994; Barr et al., 1995; Doyle et al., 1995; Wagner and Alger, 1995). Although these studies generally agree that the influence of previous synaptic activity is an important factor controlling the reversibility of a response, they differ with respect both to the time dependence and magnitude of the reversal effect and to the involvement of
A
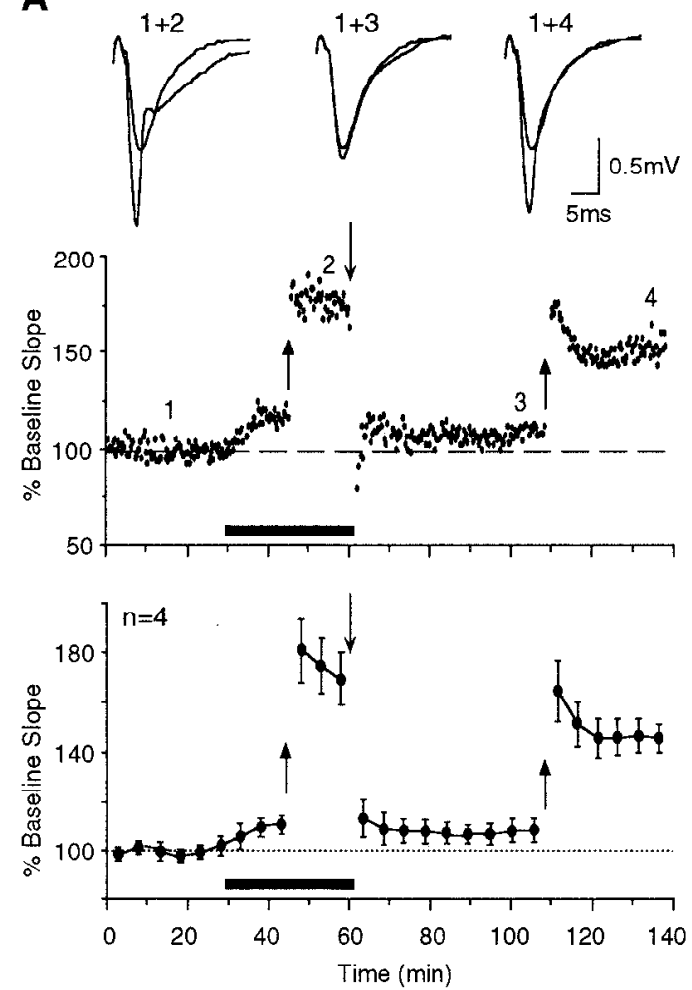

B

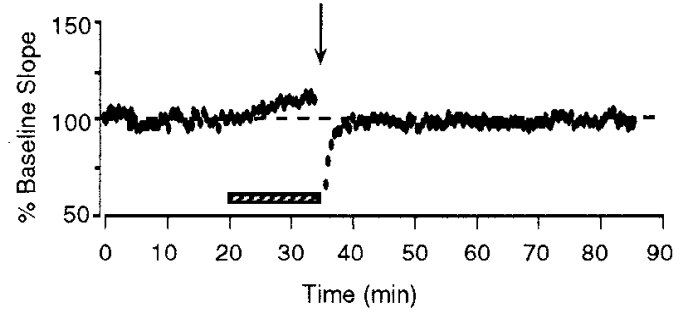

Figure 5. Reversal of LTP is reversible. $A$, Individual experiment and group data show reversal of LTP by TPS given in the presence of $500 \mu \mathrm{M}$ BDP-12 at 15 min after induction followed 60 min later by reinduction of LTP. Superimposed responses above top graph illustrate recordings taken at the times indicated by the numbers in the graph. Each data point in the botom graph represents the mean of an average of 12-16 responses/ animal. $B$, Same stimulation (TPS) that is effective in promoting LTP reversal leaves a naive pathway unaffected.

NMDA receptors. The reasons for these discrepancies are not clear but could be attributable partly to the use of stimulation patterns of variable strengths, durations, and frequencies, resulting in activation of different cellular processes that may vary in their mode of action and produce different types of depressive effects. The lack of impact on the reversal process seen with AP-5 in the present study suggests that the degree of postsynaptic depolarization produced by single pulse stimulation at $5 \mathrm{~Hz}$ was not sufficient to activate the NMDA receptor, even in the presence of BDP-12. It should be noted that BDP-12 not only incrcases cxcitatory inputs to principal cells in ficld CA1 but also facilitates the connections to feedforward inhibitory neurons known to antagonize strongly the depolarization in principal cells caused by excitatory inputs. Low-frequency stimulation at stronger intensity and/or at higher rate also might be effective at reversing LTP, but perhaps via a mechanism that involves NMDA receptors. Whatever stimulation pattern is used for LTP reversal 

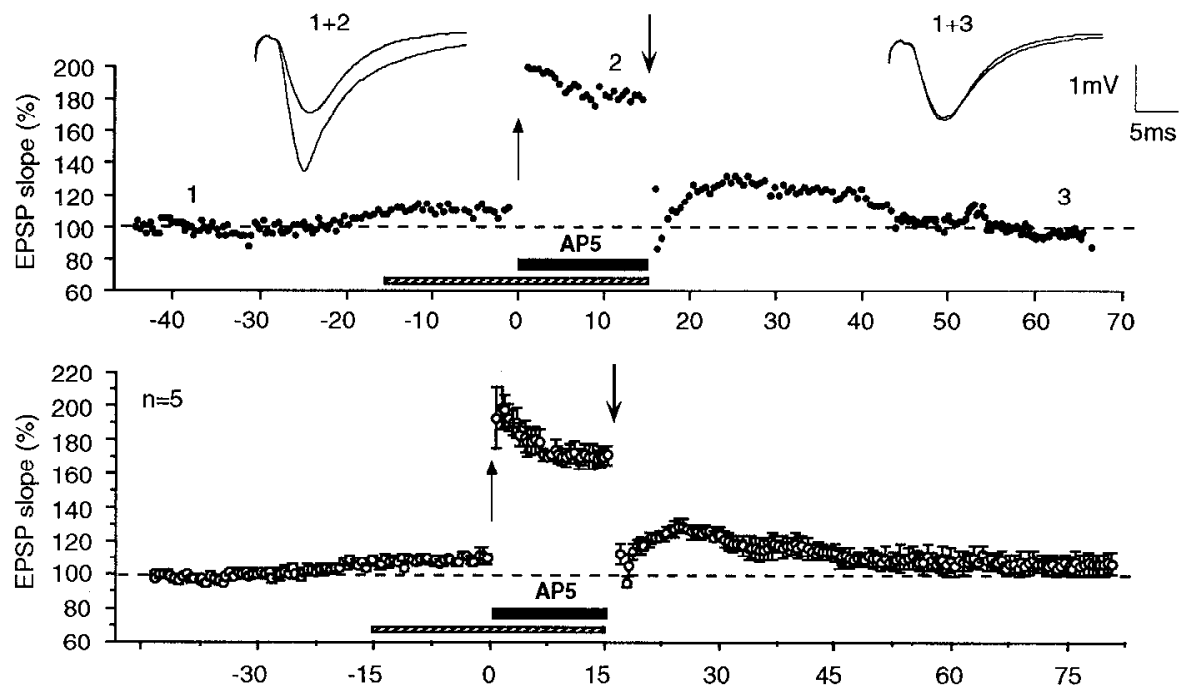

Figure 6. Reversal of LTP with a $1 \mathrm{~min}$ train of TPS does not require NMDA receptor activation. Individual experiment and group data show the failure of the NMDA receptor antagonist D,L-AP-5 $(200 \mu \mathrm{M})$ to prevent LTP reversal by TPS delivered in the presence of $500 \mu \mathrm{M}$ BDP-12.

attempts, it is crucial to verify that the cellular mechanism initiated by the reversing stimulation produces a true reversal that is selective to the potentiated synapses receiving the stimulation. This can be evaluated most conclusively in experiments in which two independent inputs are potentiated simultaneously, followed by low-frequency stimulation delivered to one input and demonstration of the absence of lasting heterosynaptic effects in the second potentiated pathway (Larson et al., 1993) (see also Fig. $4 A$ ). Unfortunately, very few studies on depotentiation have included this type of control.

How does the LTP reversal phenomenon relate to the form of homosynaptic depression known as LTD? Both LTD and reversal of LTP are synapse-specific effects and are induced optimally by stimulation patterns that do not produce LTP, i.e., by trains of low-frequency stimulation ranging between 1 and $5 \mathrm{~Hz}$. This has led to the speculation that the reversal of LTP is a form of LTD. However, the occurrence of LTD is not contingent on the time of previous LTP induction and typically requires extended trains of low-frequency stimulation lasting up to $15 \mathrm{~min}$, whereas reversal of LTP is achieved with a 1 min stimulation episode temporally coupled with LTP induction. This might be explained by assuming that recently potentiated synapses are more susceptible to depression and, therefore, that reversal of LTP is a weak version of LTD. There are other differences between the two phenomena, however, that make this explanation unlikely. First, as demonstrated in the above studies, activation of NMDA receptors is not required for LTP reversal by TPS, whereas a majority of studies agree that the NMDA receptor antagonist AP-5 blocks the induction of LTD in area CA1 of hippocampus (Dudek and Bear, 1992; Mulkey and Malenka, 1992; Kirkwood and Bear, 1994; Thiels et al., 1994); recently, we have obtained data consistent with the NMDA receptor dependence of LTD in vitro (Stäubli and $\mathbf{J i}$, in press). Second, LTD appears to be age-dependent and more reliable and robust in slices from young animals (Dudek and Bear, 1992, 1993; Mulkey and Malenka, 1992) (for review, see Bear and Malenka, 1994; Linden, 1994; Stäubli and Ji, in press), whereas reversal of LTP is obtained easily in adult rats (Barrionuevo et al., 1980; Stäubli and Lynch, 1990). Third, LTD is elicited best by low-frequency stimulation at $1 \mathrm{~Hz}$ (Bear and Malenka, 1994), whereas we and others have found that reversal of LTP is achieved optimally with $5 \mathrm{~Hz}$ stimulation delivered within a limited time after I.TP induction (Stäubli and I ynch, 1990; I arson et al., 1993; O'Dell and Kandel, 1994). Fourth, TPS does not cause depression of control synapses (Stäubli and Lynch, 1990; Larson et al., 1993; O'Dell and Kandel, 1994), in contrast to LTD, which can be obtained independent of whether the stimulated pathway is naive or potentiated (Mulkey and Malenka, 1992; Dudek and Bear, 1993; Mulkey et al., 1993). Interestingly, we have found recently that the same stimulation parameters that produce LTD in vitro do not induce LTD in area CA1 of freely moving rats (U. Stäubli and F. Xu, unpublished observations). Combined, the above findings favor the assumption that LTD and LTP reversal are distinct phenomena.

In summary, our results show that LTP produced by a stimulation pattern that mimics bursting activity observed in behaving animals is reversed best by a second, naturally occurring stimulation code temporally coupled with LTP induction. Although the present study uses a 1 min train of TPS to reverse LTP, the minimum duration of stimulation necessary to produce depotentiation is not known. If future parametric studies establish that LTP reversal can be obtained with very brief trains $(<20 \mathrm{sec})$ of TPS that approach the limit of a theta episode as it occurs in the animal during exploration, then the question of the role of LTP reversal in behavior will become intriguing; i.e., it would not be implausible in this case that the reversal effect occurs in vivo. The issue of the functional significance of the reversal is particularly interesting in connection with the pyrrolidinone compounds (e.g., BDP-12), which have been shown to facilitate the functioning of AMPA receptors, enhance memory across a variety of tasks, and promote the induction of LTP (Stäubli et al., 1994a,b). To the extent that LTP is an encoding device, the very existence of a depotentiation pattern raises the possibility that the brain has an active reversal or "forgetting" mechanism that it uses to erase information selectively and refine memories by removing potentiation in subpopulations of recently stimulated synapses, thereby sharpening representations during the memory consolidation period. 


\section{REFERENCES}

Andersen P, Sundberg SH, Sveen O, Wigstrom H (1977) Specific longlasting potentiation of synaptic transmission in hippocampal slices. Nature 266:736-737.

Arai A, Kessler M, Lynch G (1990a) The effects of adenosine on the development of long-term potentiation. Neurosci Lett 119:41-44.

Arai A, Kessler M, Xiao P, Ambros-Ingerson J, Rogers G, and Lynch G (1994) A centrally active drug that modulates AMPA receptor gated currents. Brain Res 638:343-346.

Arai A, Larson J, Lynch G (1990b) Anoxia reveals a vulnerable period in the development of long-term potentiation. Brain Res 511:353-357.

Barr DS, Lambert NA, Hoyt KL, Moore SD, Wilson WA (1995) Induction and reversal of long-term potentiation by low- and high-intensity pattern stimulation. J Neurosci 15:5402-5410.

Barrionuevo G, Schottler F, Lynch G (1980) The effects of repetitive low frequency stimulation on control and "potentiated" responses in the hippocampus. Life Sci 27:2385-2391.

Bashir ZI, Collingride GL (1994) An investigation of depotentiation of long-term potentiation in the CA1 region of the hippocampus.

Bear MF, Malenka RC (1994) Synaptic plasticity: LTP and LTD. Curr Opin Neurobiol 4:389-399.

Bittar P, Muller D (1993) Time-dependent reversal of long-term potentiation by bricf cooling shocks in rat hippocampal slices. Brain Res 620:181-188.

Doyle C, Rowan MJ, Anwyl R (1995) Low-frequency stimulation induces depotentiation of long-term potentiation, but not long-term depression, in the rat hippocampal CA1 in vivo. J Physiol (Lond) 483:68P.

Dudek SM, Bear MF (1992) Homosynaptic long-term depression in area CA1 of hippocampus and the effects of NMDA receptor blockade. Proc Natl Acad Sci USA 89:4363-4367.

Dudek SM, Bear MF (1993) Bidirectional long-term modification of synaptic effectiveness in the adult and immature hippocampus. J Neurosci 13:2910-2918.

Dunwiddie TV, Lynch G (1978) Long-term potentiation and depression of synaptic responses in the rat hippocampus: localization and frequency dependency. J Physiol (Lond) 276:353-367.

Fujii S, Saito K, Miyakawa II, Ito K, and Kato II (1991) Reversal of long-term potentiation (depotentiation) induced by tetanus stimulation of the input to CA1 neurons of guinea pig hippocampal slices. Brain Res 555:112-122.

Fujii S, Wakizaka A, Sekino Y, Kuroda Y, Ito K, Miyakawa H, Kato H (1992) Adenosine $A_{2}$ receptor antagonist facilitates the reversal of long-term potentiation (depotentiation) of evoked postsynaptic potentials but inhibits that of population spikes in hippocampal neurons. Neurosci Lett 148:148-150.

Gustafsson B, Asztely F, Hanse E, Wigstrom H (1989) Onset characteristics of long-term potentiation in the guinea-pig hippocampal CA1 region in vitro. Eur J Neurosci 1:382-394.

Hill AJ (1978) First occurrence of hippocampal spatial firing in a new environment. Exp Neurol 62:282-297.

Kirkwood A, Bcar MF (1994) Ilomosynaptic long-term depression in the visual cortex. J Neurosci 14:3404-3412.
Larson J, Lynch G (1986) Induction of synaptic potentiation in hippocampus by patterned stimulation involves two events. Science 232:985-988.

Larson J, Wong D, Lynch G (1986) Patterned stimulation at the theta frequency is optimal for the induction of hippocampal long-term potentiation. Brain Res 386:347-350.

Larson J, Xiao P, Lynch G (1993) Reversal of LTP by theta frequency stimulation. Brain Res 600:97-102.

Linden DJ (1994) Long-term synaptic depression in the mammalian brain. Neuron 12:457-472.

Lynch GS, Dunwiddie TV, Gribkoff VK (1977) Heterosynaptic depression: a post-synaptic correlate of long-term potentiation. Nature 266:737-739.

McGaugh JL (1966) Time-dependent processes in memory storage. Science 153:1351-1358.

McGaugh JL, Introini-Collison IB, Cahill LF, Castellano C, Dalmaz C, Parent MB, Williams CL (1993) Neuromodulatory systems and memory storage: role of the amygdala. Behav Brain Res 58:81-90.

Mulkey RM, Herron CE, Malenka RC (1993) An essential role of protein phosphatases in hippocampal long-term depression. Science 261:1051-1055.

Mulkey RM, Malenka RC (1992) Mechanisms underlying induction of homosynaptic long-term depression in area CAl of the hippocampus. Neuron 9:967-975.

O'Dell TJ, Kandel ER (1994) Low-frequency stimulation erases LTP through an NMDA receptor-mediated activation of protein phosphatases. Learn Memory 1:129-139.

Otto T, Eichenbaum H, Wiener SI, Wible C (1991) Learning related patterns of CA1 spike trains parallel stimulation parameters optimal for inducing hippocampal long-term potentiation. Hippocampus 1:181-192.

Stäubli U, Ji Z-X (1996) The induction of homo- vs. heterosynaptic LTD in area CA1 of hippocampal slices from adult rats. Brain Res, in press.

Stäubli U, Lynch G (1987) Stable hippocampal long-term potentiation elicited by "theta" pattern stimulation. Brain Res 435:227-234.

Stäubli U, Lynch G (1990) Stable depression of potentiated synaptic responses in the hippocampus with $1-5 \mathrm{~Hz}$ stimulation. Brain Res 513:113-118.

Stäubli U, Perez Y, Xu F, Rogers G, Ingvar M, Stone-Elander S, Lynch G (1991a) Centrally active modulators of glutamate receptors facilitate the induction of LTP in vivo. Proc Natl Acad Sci USA 91:11158-11162.

Stäubli U, Rogers G, Lynch G (1994b) Facilitation of glutamate receptors enhances memory. Proc Natl Acad Sci USA 91:777-781.

Thiels E, Barrionuevo G, Berger T (1994) Excitatory stimulation during postsynaptic inhibition induces long-term depression in hippocampus in vivo. J Neurophysiol 72:3009-3016.

Wagner JJ, Alger BE (1995) GABAergic and developmental influences on homosynaptic long-term depression and depotentiation in rat hippocampus. J Neurosci 15:1577-1586.

Wexler EM, Stanton PK (1993) Priming of homosynaptic long-term depression in hippocampus by previous synaptic activity. NeuroReport 4:591-594. 